\title{
Identification of the human PMR1 mRNA endonuclease as an alternatively processed product of the gene for peroxidasin-like protein
}

\author{
SHAN-QING GU, ${ }^{1,2,4}$ BASKAR BAKTHAVACHALU, ${ }^{1,2,4}$ JOONHEE HAN, ${ }^{1,2,4}$ DEEPAK P. PATIL, ${ }^{1,2}$ \\ YUICHI OTSUKA, ${ }^{1,2,5}$ CHITTIBABU GUDA, ${ }^{3}$ and DANIEL R. SCHOENBERG ${ }^{1,2,6}$ \\ ${ }^{1}$ Center for RNA Biology, ${ }^{2}$ Department of Molecular and Cellular Biochemistry, The Ohio State University, Columbus, Ohio 43210-1218, USA \\ ${ }^{3}$ Center for Bioinformatics and Systems Biology, Department of Genetics, Cell Biology and Anatomy, University of Nebraska Medical Center, \\ Omaha, Nebraska 68118-5145, USA
}

\begin{abstract}
The PMR1 endonuclease was discovered in Xenopus liver and identified as a member of the large and diverse peroxidase gene family. The peroxidase genes arose from multiple duplication and rearrangement events, and their high degree of sequence similarity confounded attempts to identify human PMR1. The functioning of PMR1 in mRNA decay depends on the phosphorylation of a tyrosine in the C-terminal polysome targeting domain by c-Src. The sequences of regions that are required for c-Src binding and phosphorylation of Xenopus PMR1 were used to inform a bioinformatics search that identified two related genes as potential candidates for human PMR1: peroxidasin homolog (PXDN) and peroxidasin homolog-like (PXDNL) protein. Although each of these genes is predicted to encode a large, multidomain membrane-bound peroxidase, alternative splicing of PXDNL pre-mRNA yields a transcript whose predicted product is a $57-\mathrm{kDa}$ protein with $42 \%$ sequence identity to Xenopus PMR1. Results presented here confirm the existence of the predicted 57-kDa protein, show this is the only form of PXDNL detected in any of the human cell lines examined, and confirm its identity as human PMR1. Like the Xenopus protein, human PMR1 binds to c-Src, is tyrosine phosphorylated, sediments on polysomes, and catalyzes the selective decay of a PMR1 substrate mRNA. Importantly, the expression of human PMR1 stimulates cell motility in a manner similar to that of the Xenopus PMR1 expressed in human cells, thus providing definitive evidence linking endonuclease decay to the regulation of cell motility.
\end{abstract}

Keywords: mRNA decay; endonuclease; cell motility; PMR1

\section{INTRODUCTION}

mRNA turnover is a key regulatory step in gene expression, and changes in the decay process impact diseases ranging from diabetes to cancer. For more than a decade, the prevailing view modeled the decay process in higher eukaryotes to that of Saccharomyces cerevisiae, beginning with deadenylation followed by decapping and degradation of the mRNA body by the 5'-3' exonuclease Xrn1 (Parker and Sheth 2007), with the added twist that decay could also proceed from the $3^{\prime}$ end, sometimes simultaneously with $5^{\prime}$

\footnotetext{
${ }^{4}$ These authors contributed equally to this work.

${ }^{5}$ Present address: Department of Biological sciences, Graduate School of Science, Osaka University, Osaka 560-0043, Japan.

${ }^{6}$ Corresponding author.

E-mail schoenberg.3@osu.edu.

Article published online ahead of print. Article and publication date are at http://www.rnajournal.org/cgi/doi/10.1261/rna.031369.111.
}

decay (Murray and Schoenberg 2007). Starting in the early 1980s, there were also reports describing endonuclease cleavage associated with the decay of a variety of mRNAs (for review, see Schoenberg 2011), but endonuclease decay was for years considered a minor pathway. This has changed with the advent of massively parallel sequencing, with different groups providing convincing evidence for widespread endonuclease cleavage as both a decay process and a process for generating new families of small RNAs (FejesToth et al. 2009; Kapranov 2009; Karginov et al. 2010; Mercer et al. 2010; Plessy et al. 2010).

In contrast to the pervasive evidence of endonuclease cleavage, relatively little is known about the enzymes that catalyze this process. Knockout/knockdown experiments show that some of the cleavages come from the DroshaDicer-RISC triad of enzymes (Karginov et al. 2010), but most other cleavage events have yet to be assigned to a specific enzyme or group of enzymes. Only a handful of 
bona fide mRNA endonucleases have been identified (Tomecki and Dziembowski 2010; Schoenberg 2011; Schoenberg and Maquat 2012). These are PMR1 (Yang and Schoenberg 2004), ZC3H12A (also called MCPIP1) (Matsushita et al. 2009; Mizgalska et al. 2009), APE1 (Barnes et al. 2009), SMG6 (Huntzinger et al. 2008; Eberle et al. 2009), and IRE1 (Han et al. 2009; Hollien et al. 2009).

PMR1 was originally identified as an estrogen-induced ribonuclease activity that appeared on Xenopus liver polysomes at the same time that albumin and other serum protein mRNAs disappeared following treatment with estrogen (Pastori et al. 1991). The purified ribonuclease is a $60-\mathrm{kDa}$ protein (Dompenciel et al. 1995) that was identified as a member of the peroxidase gene family (Chernokalskaya et al. 1998) and, with the recent release of the Xenopus genome, as a product of the gene for eosinophil peroxidase. Xenopus PMR1 (xPMR1) acts in the context of its translating substrate mRNA, and substrate mRNA is stabilized by mutations that interfere with its targeting to polysomes (Yang and Schoenberg 2004). Two portions of xPMR1 proved to be critical for this, a region toward the $\mathrm{N}$ terminus and a region closer to the $\mathrm{C}$ terminus. Subsequently we identified a consensus tyrosine phosphorylation site within the C-terminal polysome targeting domain, and showed that decay was inhibited by changing the key tyrosine residue here to phenylalanine or treating cells with a general tyrosine kinase inhibitor (Yang et al. 2004). This led to the identification of $\mathrm{c}-\mathrm{Src}$ as the kinase that phosphorylates XPMR1, and work done in the context of this showed that the $\mathrm{N}$-terminal polysome-targeting domain harbors a proline-rich $\mathrm{SH} 3$ binding site that is required for formation of the complex of c-Src with xPMR1 (Peng and Schoenberg 2007). When expressed in mammalian cells, xPMR1 binds to the Ena/VASP regulators of the actin cytoskeleton, and in cell motility experiments, it colocalizes with these proteins at the leading edge. Moreover, the induced expression of xPMR1 caused a twofold increase in motility of human U2OS cells, raising the possibility of a direct role for PMR1 in controlling motility through selective mRNA decay or an indirect role similar to that shown recently for $\mathrm{ZC} 3 \mathrm{H} 12 \mathrm{~A}$, which competes with Dicer processing of some microRNAs by cleaving within the terminal loop of some precursors (Suzuki et al. 2011). The search for a cDNA clone of human PMR1 (hPMR1) began soon after the Xenopus protein was identified as a member of the peroxidase gene family but, despite years of effort, met with little success. The peroxidases arose through multiple rounds of gene duplication and recombination (Zamocky et al. 2008), and high degree of sequence similarity across species and between individual peroxidases confounded all attempts at library screening. Experiments with antibodies to human eosinophil peroxidase showed no relationship between this and hPMR1, and bioinformatics analysis of all of the major peroxidases showed all of these lack one or more of the sequence elements that are required for the binding of $\mathrm{c}$-Src and for tyrosine phosphorylation of PMR1. By use of the residues that are required for binding of $\mathrm{c}-\mathrm{Src}$ and tyrosine phosphorylation as a filter, we identified hPMR1 as a product of the gene for human peroxidasin homolog (Drosophila)-like protein (PXDNL). This gene is also referred to as cardiac peroxidase, and it only appeared in the sequence databases in 2008. Humans have two closely related genes: PXDNL and a peroxidasin homolog (Drosophila, PXDN). These genes are on different chromosomes and produce different products. PXDN does not undergo alternative splicing, and the only product of this gene is a multidomain 165-kDa protein. The fully spliced form of PXDNL encodes a $164-\mathrm{kDa}$ protein; however, PXDNL also undergoes alternative splicing, the product of which is the 57-kDa hPMR1.

\section{RESULTS}

\section{Bioinformatics identification of the human form of PMR1 as a product of the gene for peroxidasin-like protein}

The bioinformatics search for hPMR1 focused on identifying members of the peroxidase gene family that retain features of $\mathrm{xPMR} 1$ shown previously to be critical for its function as an mRNA endonuclease. Key among these are the site of tyrosine phosphorylation (Yang et al. 2004); the downstream three to four amino acids, which function to define the $\mathrm{SH} 2$ domain protein that binds to phosphotyrosine (Huang et al. 2008); a proline-rich region in the $\mathrm{N}$ terminus that is required for interaction of XPMR 1 with c-Src (Peng and Schoenberg 2007); and a pair of histidine residues that participate in the active site of the enzyme (Yang and Schoenberg 2004). The initial results of this search are summarized in the MEME analysis (Bailey et al. 2009) at the top of Figure 1, which compares all 42 of the human peroxidases and their various isoforms across the region that corresponds to the tyrosine phosphorylation site of XPMR1 (boxed). Most of these have a tryptophan instead of tyrosine at the site that is phosphorylated in xPMR1, and while xPMR1 is an alternatively processed form of Xenopus eosinophil peroxidase, there are sufficient differences between this and human eosinophil peroxidase to rule this out as the source of hPMR1. These include a tryptophan at the position that is tyrosine phosphorylated in XPMR1 (Fig. 1, bottom), differences in the three to four amino acids in the sequence immediately downstream that determine $\mathrm{SH} 2$ domain binding specificity, and the absence of the proline-rich sequence that is required for binding of c-Src and subsequent phosphorylation (data not shown).

A more detailed view of these differences is shown in at the bottom of Figure 1, where some of the major peroxidases 


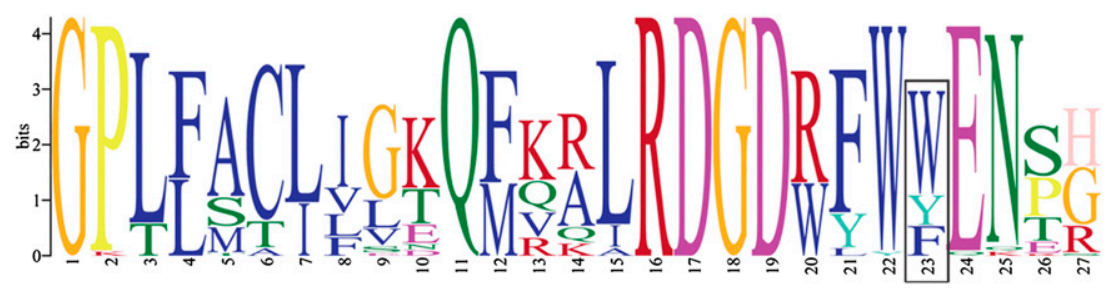

\begin{tabular}{|c|c|c|c|c|}
\hline Name & Start & p-value & Sites & \\
\hline $62865489|g b| A A Y 16985.1 \mid$ & 649 & $5.14 \mathrm{e}-36$ & AENFLPRART G LFACLI KQMKALRD GDWFWWENS VFTDAQRREL & Thyroid peroxidase \\
\hline 4557759|ref|NP_000241.1| & 657 & $2.37 e-29$ & SEPLKRKGRV G LLACIIGTQFRKLRDGDRFWWENEG VFSMQQRQAL & Myeloperoxidase \\
\hline 40549418|ref|NP_006142.1| & 624 & $3.69 \mathrm{e}-29$ & AEPLVERGRV G LLACLL GKFQQIRDGDRFWWEN G VFTNEQKDSL & Lactoperoxidase \\
\hline 119597694|gb|EAW77288.1| & 496 & $2.80 \mathrm{e}-25$ & GGLLESHGDP G LFSAIVLDQFVRLRDGDRYWFENTR NGLFSKKEIE & Dual Oxidase \\
\hline 4503595|ref|NP_000493.1] & 629 & $1.37 \mathrm{e}-22$ & AEPLLPGARV G LLACLFENQFRRARDGDRFWWQKRG VFTKRQRKAL & Eosinophil peroxidase \\
\hline 6273399|gb|AAF06354.1| & 1245 & $3.04 \mathrm{e}-26$ & VEDLVPGSRL G TLMCLLSTQFKRLRDGDRLWFEN G VFSPAQLTQI & PXDN \\
\hline $62177291 \mid$ gb|AAX70929.1| & 1211 & $6.00 \mathrm{e}-28$ & VEDIIPGTRV G TLMCLFVTQFQRLRDGDRFWEN G VFTPAQLTQL & PXDNL \\
\hline 147905582|ref|NP_001081848.1| & 627 & $9.33 e-22$ & AESLVRNGRI GKLLTCLIGNQFRRARDGDRFYEQ S VFTNEQRASI & xPMR1 \\
\hline
\end{tabular}

FIGURE 1. Comparison of amino acids surrounding the tyrosine phosphorylation site of XPMR1 with the human peroxidases. The upper panel shows a MEME analysis of the sequences of the 42 human peroxidases and their isoforms across the region corresponding to the tyrosine phosphorylation site of XPMR1 (boxed). The height of each letter corresponds to the relative representation of a given amino acid at a particular site. In the lower panel, the major human peroxidases and the peroxidasins are shown aligned with xPMR1 across the same region. The box indicates the tyrosine residue that corresponds to the phosphorylated tyrosine of xPMR1.

are aligned across the region that corresponds to the tyrosine phosphorylation site of xPMR1. The peroxidasins proved to be the only peroxidases that match xPMR1 at the tyrosine phosphorylation site (Fig. 1, bottom). The two human peroxidasin genes PXDN and PXDNL are a subfamily of the larger peroxidase gene family (Zamocky et al. 2008). These encode similar proteins of 1479 and 1463 amino acids, respectively, and their structural organization is diagrammed in Figure 2A. Both have an N-terminal signal sequence followed by six leucine-rich repeats, four immunoglobulin-like repeats, an inosine monophosphate dehydrogenase/hypoxanthine-guanosine phosphoribosyltransferase-like domain, a heme peroxidase domain, and a C-terminal von Willebrand Factor type C (vWC) domain. Only the heme peroxidase domain of these proteins is similar to XPMR1, and neither would be a candidate for hPMR1 except that alternative splicing of PXDNL generates a transcript that is predicted to encode a $57-\mathrm{kDa}$ protein that closely matches xPMR1 (Fig. 2B). In this figure, the sequence of the predicted $57-\mathrm{kDa}$ protein is aligned with that of $\mathrm{XPMR} 1$, with residues that are critical to the function of XPMR1 as an endonuclease highlighted in yellow ( $\mathrm{SH} 3$ binding site), blue (active site histidines), and red (tyrosine phosphorylation site). With the exception of a conservative substitution of asparagine for glutamine, both proteins have the same amino acids downstream from the phosphorylated tyrosine that are involved in binding by an $\mathrm{SH} 2$ domain-containing protein. Overall there is $42 \%$ sequence identity between the two proteins, and this increases to $57 \%$ if one takes into account substitution with similar amino acids.

\section{Identification of the $57-\mathrm{kDa}$ protein as the only form of PXDNL in human cell lines}

The $164-\mathrm{kDa}$ form of PXDNL was first called cardiac peroxidase based on its presence in aorta and heart. Because of this, we expected to see a $164-\mathrm{kDa}$ band and hoped to also see the $57-\mathrm{kDa}$ presumptive hPMR1 by Western blotting with antibody to the heme peroxidase domain shown in Figure 2A. Note that the locations of the epitopes that are recognized by the antibodies used here are shown in Supplemental Figure S1A. Instead, only the $57-\mathrm{kDa}$ form of PXDNL was detected in extracts from MCF-7, MDAMB-231, U2OS, and HEK293T cells (Fig. 3A) and in Huh-7 and HepG2 cells (data not shown). A faint 164-kDa band was detected in $\mathrm{K} 562$ cells, but again, the major protein was 57-kDa PXDNL. Attempts to identify a larger form of PXDNL using antibodies to the $\mathrm{N}$ - and C-terminal portions of that protein were unsuccessful (Supplemental Fig. $\mathrm{S1B}$ ), suggesting that these cells do not express mRNA for the $164-\mathrm{kDa}$ protein, or it is expressed and the protein is not translated or is rapidly degraded. RT-PCR provided evidence for exon skipping that is consistent with the 57-kDa PXDNL missing the C-terminal von Willebrand type C domain (Supplemental Fig. S2), but transcripts in which exons 19-22 are included were also seen. Some of these do not encode a functional protein, so it was not possible to reach a definitive conclusion as to the presence or absence of full-length PXDNL mRNA. Taken together, the data point to the $57-\mathrm{kDa}$ protein as the only form of PXDNL expressed in these cell lines. The $57-\mathrm{kDa}$ PXDNL was not detected in MEL cells on this particular blot; however, this 
A

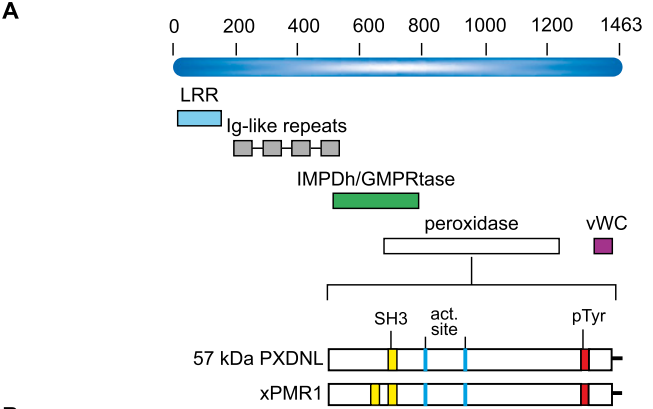

XPMR1 GQCNNRKNPILGASNTGFTRLLPVVYEDGLSVPRGWTENLPINGFPLPLARAVSNEIVRF 60

PXDNL ------------MLMHWGWFLEHDLDHTVPALSTARFSDGRPCSSVCTNDPPCFPMNTR 47 XPMR1 PNENLTLDEGRALIFMNGWTDHDLDLSPETPARSTELLEGIDCDTNCAKEPPCFPLKIP 120

PXDNL HADPRGTHAP-CMLFARSSPACASGRPSATVDSVYAREQINQQTAYIDGSNVYGSSERES 106 XPMR1 PNDPRISNQSDCIPLFRSSPVITPGSP--------VREQINILTSFIDGSQVYGSDWPLA 172

PXDNL QALRDPSVPRGLLKTGFPWPPSGKPPLLPFSTGPPTECARQEQES-_PCFLAGDHRANEHL 164 XPMR1 VKLRNNTNQLGLMATNORFTDNGLIPFLPFETAEEDFCVVTTNRSSGIPCFIGGDPRVSE QP 232

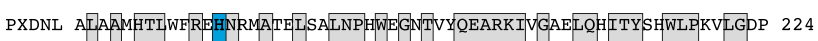
XPMR1 GLTAF ETIFVRAHNNIAARLRELNPRWSGETL YQEARKIIGGILQRITYKDWLPLLLGSE 292 PXDNL GTRMERGYRGYNPNVWNAGIINSAFATAAFREGAIILINPILYRINATLGEIS-EGHLPFHKA 283 XPMR1 MAAVEPAYRSYNESUDPRVSNVET-VVERMGEILIIAPF IYRLADGYRPLGEEPQIPLHKT 351

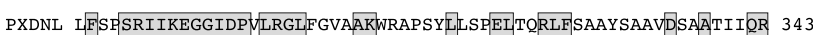
XPMR1 FENSWRVVREGGIDPYLRGLMANRAKLNRQNQLUVVDELRERLFVLFFRIGLDLTAINMQR 411

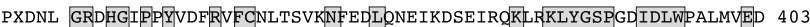
XPMR1 GREHGLPGYNAWRRECGLSAPSNYNELAAVLNNRNLAEKF IKLYGSPENIDIWVGGVAES 471

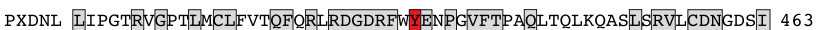
XPMR1 LURNGRIGKLLTCLI GNOFRRARDGDRFY YYEQRSVFTNEQRASIERVTLARVICDN-TKI 530

PXDNL QQVVADVFVKAE WPQDYLNCSEIPKVDIRVWQDCCAKQAGGTPEAGRVYRC 515

XPMR1 TEVPRNVFLGNRYPRDFVACSRIPTIDINPWKVA-- 564

FIGURE 2. Relationship of PXDNL to xPMR1. (A) The full-length PXDNL protein is shown schematically with relative positioning of the functional domains of the full-length protein. Beneath this, the peroxidase domain is expanded to show the relative positioning of sequence elements that are conserved between the $57-\mathrm{kDa}$ form of PXDNL and xPMR1. (B) The sequence of the 57-kDa form of PXDNL is aligned with that of the 60-kDa form of XPMR1 (xPMR60), with matching residues based on similarities with XPMR1. The sequence of xPMR1 does not start with methionine because it is generated by proteolytic cleavage from a larger precursor. In this figure, identical amino acids are shaded, the binding site for c-Src is shown in yellow, the active site histidines are shown in blue, and the tyrosine phosphorylation site is shown in red.

may be a feature of the antibody since it was raised against the human protein and a faint $57-\mathrm{kDa}$ band is observed when more extract is loaded onto the gel. Under no circumstances was there any evidence for any form of PXDNL in Cos-1 cells, a finding that is consistent with earlier work using antibodies against XPMR1, with which we found no evidence for any cross-reacting protein (Yang and Schoenberg 2004).

To determine if the $57-\mathrm{kDa}$ protein detected with the PXDNL antibody is the same as that which is predicted from sequence data, Cos-1 cells were transfected with a plasmid expressing the sequence of the predicted protein with an $\mathrm{N}$ terminal Flag tag and a C-terminal peptide that is quantitatively biotinylated in vivo (Tagwerker et al. 2006). The input sample and proteins that were recovered on streptavidin paramagnetic beads or by immunoprecipitation with antiPXDNL antibody or nonimmune IgG were then analyzed by Western blotting with an anti-Flag antibody. One-tenth of the input sample and streptavidin-bound samples were applied to lanes 1 and 2, and the similar signal intensity of these bands indicated this affinity recovery approach is highly effective. The recovery of Flag-tagged 57-kDa PXDNL with anti-PXDNL antibody but not with nonimmune IgG confirmed that the protein expressed in these cells is the same as that predicted from the sequence databases.

\section{Polysome profile analysis of 57-kDa PXDNL}

Sucrose gradient sedimentation was used to determine if 57-kDa PXDNL binds to polysomes in a manner similar to that of XPMR1 (Yang and Schoenberg 2004). The top panel of Figure 4A shows the absorbance profile for cytoplasmic extract of MCF-7 cells separated on a $10 \%-50 \%$ sucrose gradient. Beneath this is a Western blot probed with antiPXDNL antibody. Note that every two fractions were pooled prior to electrophoresis. Similar to results seen in Xenopus hepatocytes (Cunningham et al. 2001), most of the 57-kDa PXDNL sedimented with polysomes and a portion was also present in the lighter mRNP-containing fractions at the top of the gradient. To confirm that the heavier-
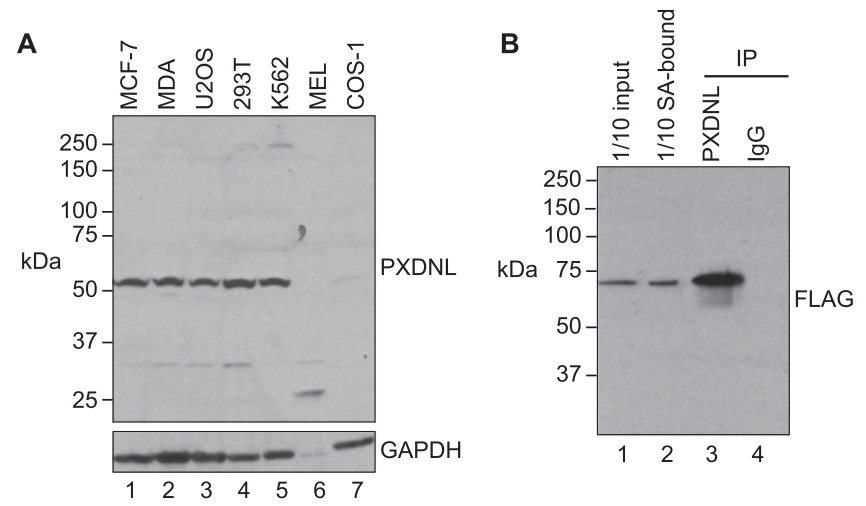

FIGURE 3. Identification of $57-\mathrm{kDa}$ PXDNL in different cell lines. (A) Thirty micrograms of cytoplasmic protein from MCF-7, MDAMB-231, U2OS, HEK-293T, K562, MEL, and Cos-1 cells was separated on a 10\% SDS-PAGE gel. A Western blot of this was probed first with an affinity-purified antibody to the peroxidase domain of human PXDNL (upper panel) and then stripped and probed with antibody to human GAPDH (lower panel) as a loading control. Note that the anti-human antibodies react poorly with both murine proteins (lane 6 ). (B) Cos-1 cells were transiently transfected with a plasmid expressing $57-\mathrm{kDa}$ PXDNL with an N-terminal Flag tag and a C-terminal sequence that is biotinylated in vivo. Note that these two tags increase the size of the recombinant protein over endogenous 57-kDa PXDNL. Cytoplasmic extract was recovered with streptavidin paramagnetic beads (lane 2) or by immunoprecipitation with anti-PXDNL antibody (lane 3) or nonimmune IgG (lane 4), and a Western blot of input and bound proteins was probed with antibody to the Flag tag. The same relative amount of input and streptavidinrecovered protein (1/10th of total sample) and $100 \%$ of the antibody bound samples were applied to the gel. 
A

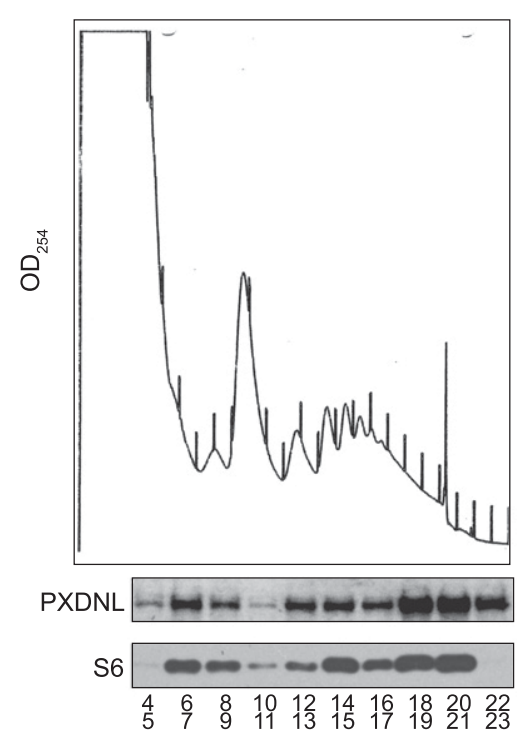

C

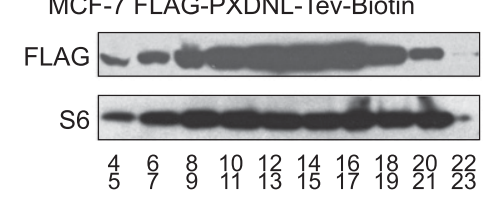

E Cos-1 FLAG-PXDNL

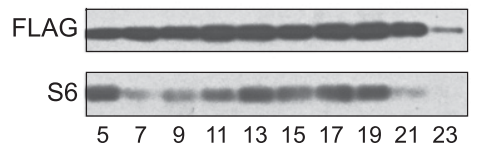

FIGURE 4. Polysome profile analysis of 57-kDa PXDNL. (A) Cytoplasmic extract from MCF-7 cells was separated on a 10\%-50\% sucrose gradient. The upper panel shows the absorbance at $254 \mathrm{~nm}$ during fractionation. Every two fractions starting with fraction 4 were pooled and ethanol precipitated, and endogenous protein was identified by Western blotting with antiPXDNL antibody or antibody to ribosomal protein S6. (B) MCF-7 cells were treated with puromycin for $15 \mathrm{~min}$ prior to lysis and analyzed as in $A$. (C) Tetracycline repressor was introduced by viral transduction into MCF-7 cells. These were stably transfected with a tetracycline-inducible plasmid expressing the same form of PXDNL as in Figure 3B with an N-terminal Flag tag and a C-terminal sequence that is biotinylated in vivo. Cells were induced for $6 \mathrm{~h}$ with doxycycline, and cytoplasmic extract was analyzed on a linear $10 \%-50 \%$ sucrose gradient as in $A$ with the exception that Western blotting of the recovered gradient fractions was performed using anti-Flag monoclonal antibody and with antibody to ribosomal protein S6. (D) The same cells that were used in $C$ were treated for 15 min with puromycin prior to lysis and analyzed as in $C$. (E) Cos-1 cells were transiently transfected with a plasmid expressing 57-kDa PXDNL with just an N-terminal Flag tag. Cytoplasmic extract was separated on a linear $10 \%-50 \%$ sucrose gradient as in $A$ and $C$, and protein recovered in odd-numbered fractions was detected by Western blotting with anti-Flag monoclonal antibody.

We next examined the sedimentation behavior of recombinant $57-\mathrm{kDa}$ PXDNL. A line of MCF-7 cells with inducible expression of $57-\mathrm{kDa}$ PXDNL was generated by stable transfection with plasmids expressing the tetracycline repressor protein and the Flag-tagged form of PXDNL used in Figure 3B under control of a tetracycline-inducible promoter. The overall sedimentation pattern of Flag-57$\mathrm{kDa}$ PXDNL is similar to that of the endogenous protein except for a slight shift to lighter polysome fractions (Fig. $4 \mathrm{C})$. We suspect this may be due to overexpression of the recombinant protein. As seen for endogenous $57-\mathrm{kDa}$ PXDNL in Figure 4B, treating cells with puromycin prior to lysis shifted the Flag-tagged protein to lighter fractions (Fig. 4D), confirming that recombinant 57-kDa PXDNL binds to polysomes.

Our initial work with recombinant xPMR1 used transfection into Cos-1 cells because there was no evidence in these cells for a protein that cross-reacted with anti-xPMR1 antibody (Yang and Schoenberg 2004). To complete the analysis of sedimentation behavior, polysome profile analysis was performed on Flag-57-kDa PXDNL expressed in transiently transfected Cos-1 cells (Fig. 4E). The overall pattern was similar to those seen in Figure 4, A and C, thus confirming that the protein expressed in transiently transfected cells binds to polysomes in a manner similar to that of XPMR1 and endogenous 57-kDa PXDNL.

\section{7-kDa PXDNL binds to c-Src and is tyrosine phosphorylated}

The targeting of xPMR1 to polysomes requires phosphorylation of a tyrosine (Y650) in the C-terminal polysometargeting domain of the protein (Yang et al. 2004). Tyrosine kinases bind directly to their substrates, and the re-

sedimenting population of $57-\mathrm{kDa}$ PXDNL was bound to polysomes, cells were treated with puromycin prior to lysis. The effectiveness of puromycin in dissociating polysomes is shown by the absorbance trace in the upper panel of Figure $4 \mathrm{~B}$ and by Western blotting with an antibody to ribosomal protein S6. Puromycin treatment also shifted the sedimentation of $57-\mathrm{kDa}$ PXDNL to the lighter gradient fractions, a result that is consistent with its binding to polysomes. covery of c-Src with xPMR1 was key to its identification as the kinase that is responsible for its phosphorylation (Peng and Schoenberg 2007). To determine if the same holds true for the $57-\mathrm{kDa}$ PXDNL a plasmid expressing Flag-tagged 57-kDa PXDNL with a C-terminal biotinylated peptide sequence was transfected together with the empty vector or plasmids expressing constitutively active (CA-Src) or dominant-negative forms (DN-Src) of c-Src into the line of 
Src-deficient Cos-1 cells that was used in our previous study on c-Src (Peng and Schoenberg 2007). Although the same amount of each of the Src-expressing plasmids was transfected into these cells, expression of DN-Src was routinely greater than CA-Src (Fig. 5, lanes 2 and 3). Biotinylated $57-\mathrm{kDa}$ PXDNL and its associated proteins were recovered with streptavidin paramagnetic beads and analyzed by Western blotting with antibodies to the Flag tag on the $57-\mathrm{kDa}$ PXDNL, to $\mathrm{c}-\mathrm{Src}$, and to phosphotyrosine (PY20) (Fig. 5). Similar to results observed with XPMR1 (Peng and Schoenberg 2007), both active and inactive forms of $\mathrm{c}$-Src were recovered with the $57-\mathrm{kDa}$ PXDNL, and tyrosine phosphorylated 57-kDa PXDNL and c-Src were recovered in cells expressing the active form of the kinase (lane 5). Note that the phosphotyrosine signal on c-Src results from its autophosphorylation, and the Flag and biotinylation tags added to the 57-kDa PXDNL are responsible for the increased size.

\section{Activity assays confirm the identity of 57-kDa PXDNL as hPMR1}

Albumin mRNA has proven to be a reliable substrate for measuring PMR1 activity, and as a final demonstration that the 57-kDa PXDNL is hPMR1, we examined its ability to selectively degrade this, but not luciferase mRNA (Yang and Schoenberg 2004). Triplicate cultures of U2OS cells were cotransfected with plasmids expressing albumin and luciferase (control) mRNA together with plasmids expressing GFP, xPMR1, or $57-\mathrm{kDa}$ PXDNL and assayed by RNase protection (Fig. 6A) as in the method described by Yang and Schoenberg (2004). The selective loss of albumin mRNA by 57-kDa PXDNL (lanes 10-12) supports the identification of this protein as hPMR1. Confirming evidence for this is in Figure 6B, where tetracycline repressor-expressing MCF-7

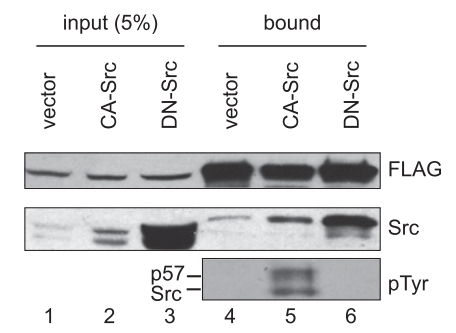

FIGURE 5. The 57-kDa PXDNL binds to c-Src and is tyrosine phosphorylated. The line of Src-deficient cells used previously to characterize the interaction of XPMR1 with c-Src (Peng and Schoenberg 2007) was cotransfected with a plasmids expressing Flag-57-kDa PXDNL with a C-terminal biotinylated tag and empty vector (lanes 1,4 ), constitutively active c-Src (CA-Src; lanes 2,5), or a dominant-negative form of c-Src (DN-Src; lanes 3,6), and cytoplasmic extracts from each of the transfectants were recovered with streptavidin paramagnetic beads. Five percent of input (lanes 1-3) and $100 \%$ of bound material (lanes 4-6) was applied to a 10\% SDS-PAGE gel and analyzed by Western blotting with antibodies to the Flag tag, to c-Src, and to phosphotyrosine (pTyr).
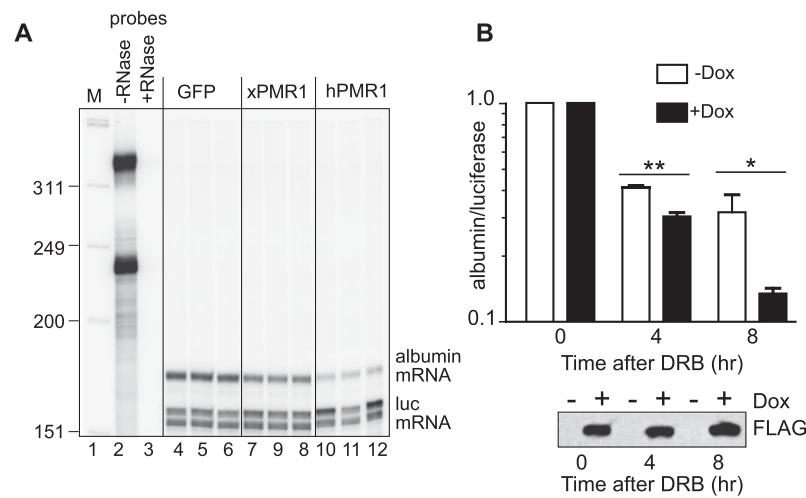

FIGURE 6. Identification of 57-kDa PXDNL as hPMR1. (A) Triplicate cultures of U2OS cells were transfected with plasmids expressing GFP (lanes 4-6), xPMR1 (lanes 7-9), or 57-kDa PXDNL (lanes 10-12) together with plasmids expressing albumin (substrate) and luciferase (control) mRNA. Cytoplasmic RNA recovered from each transfectant was analyzed by RNase protection assay. Lanes 2 and 3 contain the probes without $(-)$ or with RNase $(+)$ treatment. (B) Triplicate cultures of tetracycline-inducible MCF-7 cells at 30\%-40\% confluency were cotransfected with $0.2 \mu \mathrm{g}$ each of plasmids expressing Xenopus albumin mRNA, luciferase mRNA, and $0.75 \mu \mathrm{g}$ of pcDNA4/TO-FlaghPMR1-Tev-biotin (57-kDa PXDNL). The cells were cultured for $18 \mathrm{~h}$ without ( - Dox) or with (+Dox) doxycycline, and DRB was added at time $=0$ to block transcription. Cytoplasmic RNA isolated at 0,4 , and $8 \mathrm{~h}$ was analyzed by quantitative RT-PCR, and albumin mRNA was normalized to luciferase mRNA. The mean value at time 0 for albumin normalized to luciferase RNA was arbitrarily set to 1 . Shown are the mean \pm SD from triplicate cultures; ${ }^{\star} P<.05,{ }^{* *} P<.001$ by Student's $t$-test. The lower panel is a Western blot of cytoplasmic protein recovered from cultures treated \pm doxycycline at each time point probed with antibody to the Flag tag on hPMR1.

cells were transfected with $57-\mathrm{kDa}$ PXDNL under a tetracycline-regulated promoter, albumin mRNA, and luciferase mRNA. Half of the cultures received doxycycline to induce protein expression, and 5,6-dichlorobenzimidazole 1- $\beta$-Dribofuranoside (DRB) was added at time 0 to block transcription. Changes in albumin and luciferase mRNA were determined by quantitative RT-PCR performed on cytoplasmic RNA that was isolated at the time of DRB addition (time 0 ) and at 4 and $8 \mathrm{~h}$ after addition of DRB. The accelerated loss of albumin mRNA in doxycycline-treated cells confirms that $57-\mathrm{kDa}$ PXDNL is hPMR1.

\section{Stabilization of substrate mRNA following knockdown of hPMR1}

Two approaches to knocking down hPMR1 were used to determine if the endogenous protein is responsible for the loss of albumin mRNA in cells that were not treated with doxycycline to induce expression of recombinant protein. In the first of these, lenitviral transduction of an shRNA against hPMR1 was used to generate a line of U2OS cells with reduced hPMR1. These cells expressed $\sim 60 \%$ less hPMR1 than did nontransduced cells or cells that were transduced with a scrambled control shRNA (Fig. 7A). 

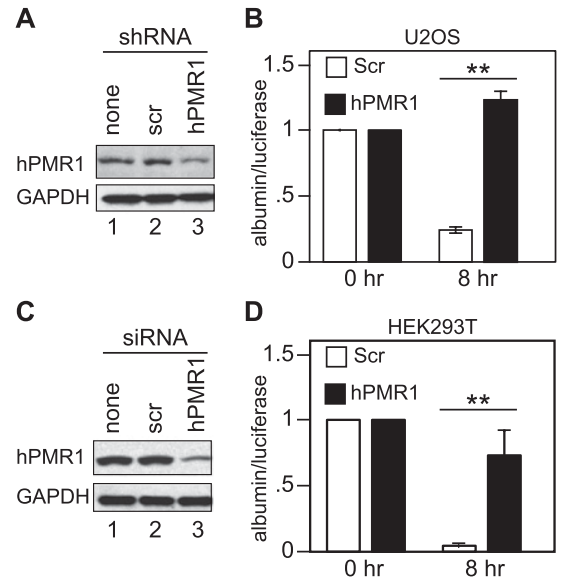

FIGURE 7. Impact of hPMR1 knockdown on albumin mRNA decay. (A) U2OS cells were transduced with lentivirus expressing a scrambled control shRNA (scr; lane 2) and shRNA against hPMR1 (lane 3). Knockdown efficiency was determined by Western blotting with an anti-PXDNL (hPMR1) antibody (upper panel) or antibody to GAPDH (lower panel). (B) Triplicate cultures of the cell lines transduced with the scrambled shRNA (open bars) or hPMR1 shRNA (filled bars) were transfected with albumin and luciferase reporter plasmids $16 \mathrm{~h}$ prior to adding DRB to block transcription. Cytoplasmic RNA recovered at the time of DRB addition $(t=0)$ and $8 \mathrm{~h}$ after DRB was analyzed by quantitative RT-PCR, as in Figure 6B. (C) Triplicate cultures of HEK293T cells were transfected twice at 24-h intervals with siRNA targeting hPMR1 or a scrambled control, and knockdown efficiency was determined by as in $A$. (D) Plasmids expressing albumin and luciferase mRNA were added to the second round of siRNA transfection, followed $16 \mathrm{~h}$ later by addition of DRB. Cytoplasmic RNA recovered at the time of DRB addition and $8 \mathrm{~h}$ later was assayed as in $B$. In $B$ and $D$, the mean value at time 0 for albumin mRNA normalized to luciferase mRNA was arbitrarily set to 1 ; error bars, $\mathrm{SD}$; ${ }^{* *} P<.001$ by Student's $t$-test.

Similar to the approach used in Figure $6 \mathrm{~B}$, triplicate cultures were transfected with albumin and luciferase reporters and treated with DRB to block transcription. Cytoplasmic RNA was recovered at the time of DRB addition and $8 \mathrm{~h}$ later, and albumin and luciferase mRNA were assayed by quantitative RT-PCR. Over this interval, albumin mRNA declined significantly in cells transduced with the scrambled control (Fig. 7B), whereas there was essentially no loss of albumin mRNA in cells that were transduced with shRNA targeting hPMR1.

To confirm that the stabilization of albumin mRNA resulted from reduced hPMR1 and not from something that might have changed during the selection process, this experiment was repeated using HEK293T cells that were transfected with an siRNA targeting the same sequence on hPMR1. Knockdown was more efficient, reducing the amount of hPMR1 to $20 \%$ of control (Fig. 7C), and again, this resulted in the stabilization of albumin mRNA (Fig. 7D), although not to the extent seen in shRNA-expressing cells. These data confirm that endogenous hPMR1 is responsible for the loss of albumin mRNA in Figure $6 \mathrm{~B}$ and, together with the data in Figure 6, confirm that the $57-\mathrm{kDa}$ form of PXDNL is hPMR1.

\section{Stimulation of cell motility by induced expression of hPMR1}

In previous work using a monolayer "wound healing" assay to study cell motility, we showed that induced expression of active XPMR1 in human cells caused a twofold increase in motility, whereas an inactive form of the enzyme had no impact on motility (Peng et al. 2009). Until now the biological relevance of that observation remained in question because that study used the frog protein expressed in human cells. This has now been confirmed by a similar experiment with cells expressing hPMR1 (Fig. 8). In this experiment, the cells used for polysome profile analysis in Figure 4, C and D, were grown until almost confluent before doxycycline was added to half of the cultures to induce expression of Flag-hPMR1 (i.e., 57-kDa PXDNL). Four hours prior to the start of the experiment, Mitomycin $\mathrm{C}$ was added to block replication, and the same three fields from triplicate cultures of each treatment group were photographed over a period of $32 \mathrm{~h}$ after scoring the monolayer. The plot of gap closure as a function of time (left panel) shows that induced expression of hPMR1 caused a twofold increase in cell motility, similar to that seen previously with XPMR1 in human cells, and Western blotting with anti-Flag antibody confirmed the expression of hPMR1 in doxycycline-treated cells. While the precise targets have yet to be determined, these data provide convincing evidence for the involvement of endonuclease decay in controlling cell motility.

\section{DISCUSSION}

xPMR1 cDNA was sequenced in 1998 (Chernokalskaya et al. 1998), but despite numerous attempts, only now were

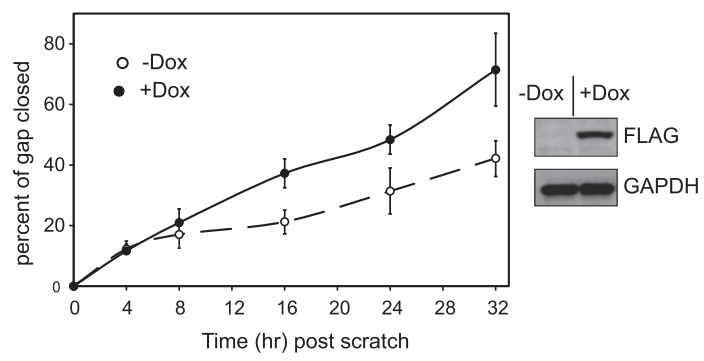

FIGURE 8. hPMR1 stimulation of cell motility. Triplicate cultures of MCF-7 cells that were stably transfected with plasmids expressing tetracycline repressor protein and Flag-hPMR1 under a tetracyclineinducible promoter were treated for $24 \mathrm{~h} \pm$ doxycycline prior to scoring the monolayer. Mitomycin $\mathrm{C}$ was added $4 \mathrm{~h}$ prior to the start of the motility experiment to block replication, and the same three fields from each set of cultures were photographed at each time point. Changes in the distance across the gap were used to calculate the percentage of closure as a function of time, and the data represent the mean \pm SD obtained at each time point; differences between treatment groups were determined by Student's $t$-test or two-way ANOVA. There was no difference at times 0,4 , and $8 \mathrm{~h}$, but at later time points, differences in the degree of gap closure were statistically significant, with $P<.0001$. The right panel is a Western blot of protein recovered at the end of the experiment probed with anti-Flag antibody. 
we successful in identifying hPMR1. In hindsight this was made particularly challenging by the size and complexity of the peroxidase gene family, the high degree of sequence similarity among the different peroxidases, and the late appearance in sequence databases (i.e., 2008) of the gene that encodes the human protein. Compounding this was the fact that the human and frog proteins are products of different genes. Whereas XPMR1 is an alternatively processed form of Xenopus eosinophil peroxidase, hPMR1 arises from alternative splicing of the pre-mRNA encoded by the gene for peroxidasin-like protein. While we do not know the reason why PMR1 is encoded by different genes in these species, the most likely explanation appears to be exon shuffling during the numerous recombination events that occurred in the expansion of the peroxidase gene family.

The human genome encodes two peroxidasins, PXDN and PXDNL. PXDN was identified as a p53-responsive gene (Horikoshi et al. 1999) and has been detected in the endoplasmic reticulum of skin and lung fibroblasts (Peterfi et al. 2009). It also accumulates in the extracellular matrix and peritubular space in a mouse model of kidney fibrosis, suggestive of a role in fibrotic responses. PXDNL was originally identified as vascular peroxidase because of the relatively high levels of immunoreactive protein in heart and vasculature (Cheng et al. 2008). Little else is known about the biochemical or functional properties of either of these proteins. Both are predicted to encode large $(\sim 164$ $\mathrm{kDa}$ ) proteins with six N-terminal leucine-rich repeats, four immunoglobulin-like repeats, a phosphoribosyltransferase-like domain, a heme peroxidase domain, and a C-terminal vWC domain. There is no evidence for alternative splicing of PXDN transcripts, so the full-length protein is the only product of this gene, but PXDNL is alternatively spliced; in fact, the $57-\mathrm{kDa}$ form of PXDNL is the only form that is detected in most human cell lines by Western blotting with affinity purified antibody to the heme peroxidase domain. While we cannot exclude the possibility that these cells express mRNA for the full-length protein (Supplemental Fig. S2), there is no evidence for this by Western blotting with antibodies against the $\mathrm{N}$ - and C-terminal portions of 164-kDa PXDNL (Supplemental Fig. S1).

hPMR1 has all of the features found to be essential for xPMR1 to function as an mRNA endonuclease (Fig. 2B). These include the N-terminal proline-rich $\mathrm{SH} 3$ sequence that is required for its binding to and phosphorylation by c-Src (Peng and Schoenberg 2007), and the tyrosine phosphorylation site and adjacent amino acids that define an $\mathrm{SH} 2$ binding site. Besides these critical features that distinguish hPMR1 from PXDN, the positioning of active site histidine residues is conserved between XPMR1 and hPMR1, and changing these to alanine inactivates the endonucleolytic activity of both xPMR1 (Yang and Schoenberg 2004) and hPMR1 (data not shown). hPMR1 sediments with polysomes (Fig. 4), binds to and is phosphorylated by
c-Src (Fig. 5), and catalyzes the degradation of a canonical PMR1 substrate mRNA (Figs. 6, 7).

The identification of hPMR1 represents a major advance in studying endonuclease-mediated mRNA decay. Although it is now clear that endonucleolytic cleavage has a broad impact on the mRNA transcriptome (Karginov et al. 2010; Mercer et al. 2010), only a handful of bona fide mRNA endonucleases have been identified (for review, see Schoenberg 2011). The ability to study PMR1 in a homologous system opens up new areas of investigation, particularly as we move forward with the identification of its mRNA targets. An example of an advance made possible by the identification of hPMR1 comes from work on the regulation of parathyroid hormone (PTH). The steadystate level of PTH mRNA is controlled by the binding of KSRP and AUF1 to an AU-rich element in the 3' UTR in response to changes in levels of calcium or phosphate. Nechama et al. (2009) showed that the regulated decay of PTH mRNA involves endonuclease cleavage by xPMR1 through a process that involves interaction of the endonuclease with KSRP and the exosome. Given the similarity between xPMR1 and hPMR1, it is likely that results obtained with the frog enzyme are biologically meaningful and support a central role for endonuclease decay in calcium homeostasis. Another example comes from our work linking hPMR1 to cell motility (Fig. 8). PMR1 stimulation of cell motility was first seen using human cells expressing xPMR1 (Peng et al. 2009), but without knowledge of the human protein, the question remained as whether this, too, was a biologically relevant finding. This question is now resolved, opening up a new aspect of mRNA decay as it relates to motility and perhaps to the invasive growth of malignant cells.

Endonucleases were long considered minor contributors to the overall decay process, but this view is changing with evidence for cleavage throughout the mRNA transcriptome (Karginov et al. 2010; Mercer et al. 2010). In addition to generating truncated mRNAs with re-capped $5^{\prime}$ ends (Schoenberg and Maquat 2009), endonuclease cleavage events create new families of small RNAs whose function have yet to be determined (Fejes-Toth et al. 2009; Plessy et al. 2010), and have recently been shown to function in regulating microRNAs by cleaving within the unpaired loop of pre-miRs (Suzuki et al. 2011). The identification of hPMR1 makes it possible now to identify its native substrate mRNAs and determine if hPMR1 has a broader role in post-transcriptional processes beyond its function in mRNA decay.

\section{MATERIALS AND METHODS}

\section{Cell culture}

MCF-7, MDA-MB-231, U2OS, HEK-293T, K562, MEL, and Cos-1 cell lines were obtained from ATCC. MCF-7 cells were grown in 
RPMI-1640 medium (Invitrogen) supplemented with 10\% FBS, $2 \mathrm{mM}$ L-glutamine, $1.0 \mathrm{mM}$ sodium pyruvate, and $10 \mathrm{mM}$ Hepes and $4.5 \mathrm{~g} / \mathrm{L}$ glucose. MDA-MB-231 cells were grown in DMEM medium (Invitrogen) supplemented with $4 \mathrm{mM}$ L-glutamine and 10\% FBS, and U2OS, HEK-293T, K562, MEL, and Cos-1 cells were grown in McCoy's 5A medium (Invitrogen) plus 10\% FBS. A tetracycline-inducible line of MCF-7 cells was generated by transfection with $\mathrm{pcDNA6/TR}$ (Invitrogen) and selection in medium containing $6 \mu \mathrm{g} / \mathrm{mL}$ blasticidin. To generate a stable line expressing epitope-tagged hPMR1, these cells were transfected with pcDNA4/TO-Flag-hPMR1-Tev-biotin and selected for $5 \mathrm{~d}$ in medium containing $600 \mu \mathrm{g} / \mathrm{mL}$ Zeocin followed by $1 \mathrm{wk}$ in Zeocin-free medium. Tetracycline-inducible expression was confirmed by Western blotting with an anti-Flag antibody. The impact of hPMR1 on cell motility was determined using the previously described wound healing assay (Peng et al. 2009).

\section{Cell motility assay}

The $2.5 \times 10^{5}$ MCF-7 cells that were stably transfected with pcDNA4/TO-Flag-hPMR1-Tev-biotin (see above) were plated in six-well plates. After reaching $70 \%-80 \%$ confluence, hPMR1 expression was induced by adding $0.5 \mu \mathrm{g} / \mathrm{mL}$ doxycycline to one-half of the plates, and these were maintained for $24 \mathrm{~h}$. Replication was blocked by adding Mitomycin $\mathrm{C}$ to the cultures $4 \mathrm{~h}$ prior to scoring the monolayer with a $1000-\mu \mathrm{L}$ pipette tip. The plates were washed two times with PBS to remove nonadherent cells, and the gap in the monolayer was visualized under phase contrast using a Nikon Eclipse Ti microscope with a programmable stage. The use of a computer-controlled microscope and stage insured that the same fields were photographed at each time point. The relative rate of cell migration was determined as the percentage of the gap closed as a function of time for three locations each in triplicate cultures. These data were used to determine the mean \pm SD plotted in Figure 8. Data reduction was performed using NIS-Elements AR software, and differences between treatment groups at each time point were analyzed by Student's $t$-test or by two-way ANOVA. There was no statistical difference during the first $8 \mathrm{~h}$; however at later times, the degree of gap closure between the groups was statistically significant, with $P<.0001$.

\section{Plasmid constructs}

A cDNA corresponding to $57-\mathrm{kDa}$ PXDNL was obtained from Open Biosystems (catalog no. MHS 4426-99240274). The insert was PCR amplified with primers containing EcoRI and NotI restriction sites, cleaved with these enzymes, and inserted in place of GFP in pcDNA3-myc-GFP (Yang and Schoenberg 2004) to create pcDNA3-myc-hPMR1 (57-kDa PXDNL) and pcDNA4/TOmyc-hPMR1. To generate the Flag-tagged protein (pcDNA3-FlaghPMR1), the myc tag was removed by digesting with KpnI and SfiI and replacing this with a double-stranded DNA fragment consisting of 5'-GGTACCATGGACTACAAGGACGACGATGA CAAGATGGCCATGGAGGCC annealed to $5^{\prime}$-GGCCTCCATGGCCA TCTTGTCATCGTCGTCCTTGTAGTCCATGGTACC that was digested with the same enzymes. This insert was inserted into pcDNA4/TO to create the tetracycline-inducible Flag-tagged protein (pcDNA4/TO-Flag-hPMR1). For high-efficiency affinity purification, this was further modified by addition of a C-terminal tag containing a site for cleavage by $\mathrm{Tev}$ protease and a peptide that is biotinylated in vivo (Tagwerker et al. 2006). The plasmid pFA6a-HTB-hphMX4 containing this sequence was provided by Dr. Peter Kaiser (University of California, Irvine). The region containing the Tev cleavage site and biotin tag was PCR amplified with forward primer 5'-AATC TAGCGGCCGCGGAGAATCTGTACTTTCAATCA and reverse primer 5' -ATTACAGGGCCCTCATTAAACGCCGCCGATCTTGAT to generate a double-stranded DNA with a $5^{\prime}$ NotI site and a $3^{\prime}$ ApaI site. This was inserted into pcDNA4/TO-Flag-hPMR1 that was digested with the same enzymes to generate pcDNA4/TOFlag-hPMR1-Tev-biotin. All constructs were confirmed by DNA sequencing.

\section{Antibodies}

Affinity-purified rabbit polyclonal antibody to the PXDNL peroxidase domain and mouse monoclonal antibodies to GAPDH and Flag were obtained from Sigma-Aldrich. The PXDNL antibody was raised against a fragment of human PXDNL (Entrez Gene identification no. 137902) corresponding to amino acids $1078-1209$ of the full-length form and $277-408$ in the $57-\mathrm{kDa}$ form of PXDNL. Mouse anti-myc monoclonal antibody (9E10), immobilized anti-myc monoclonal antibody, c-Src (B-12), ribosomal protein S6 (E13), horseradish peroxidase-coupled goat antimouse IgG, and goat anti-rabbit IgG were obtained from Santa Cruz Technology. Phosphotyrosine mouse monoclonal antibody PY20 was from BD Biosciences.

\section{shRNA knockdown of hPMR1 in U2OS cells}

pGIPZ lentiviral vectors harboring hairpin sequence from V2LHS17936 (denoted hPMR1) was obtained from Open Biosystems. pLKO.1 scrambled control hairpin lentiviral vector was obtained from Addgene (no. 1864). The lentiviral particles were packaged by transfecting HEK293T cells with each of these vectors with Addgene third-generation packaging plasmids pRSV-Rev (ID:12253) and $\mathrm{pMDlg} / \mathrm{pRRE}(\mathrm{ID}: 12251)$ together with envelope plasmid pMD2.G (no. 12259) using Fugene 6. Supernatants were harvested 48 and $72 \mathrm{~h}$ post-transfection and passed through $0.45-\mu \mathrm{m}$ filter to remove debris. The particles were concentrated by ultracentrifugation at $107,000 \mathrm{~g}$ for $1.5 \mathrm{~h}$ at $4^{\circ} \mathrm{C}$ in a Sorvall $\mathrm{TH} 641$ rotor and stored at $-80^{\circ} \mathrm{C}$. For viral transduction, $2.5 \times 10^{5} \mathrm{U} 2 \mathrm{OS}$ cells were inoculated in a single well of a six-well dish with increasing amounts of each supernatant, and $2 \mu \mathrm{g} / \mathrm{mL}$ of puromycin was added $24 \mathrm{~h}$ later. Transduced cells were selected for $3 \mathrm{~d}$ in puromycincontaining medium, followed by an additional $3 \mathrm{~d}$ in puromycin-free medium, and individual clones were analyzed by Western blotting with antibodies to PXDNL and GAPDH to determine the efficiency of knockdown.

\section{siRNA knockdown of hPMR1 in HEK-293T cells}

A 21-nucleotide (nt) siRNA custom duplex with sense sequence 5'-CAGAAUACCCACAGGAUUAUU- $3^{\prime}$ and antisense sequence 5'-UAAUCCUGUGGGUAUUCUGUU-3' was purchased from Thermo/Dharmacon. This corresponds to positions 3925-3946 of full-length PXDNL mRNA and 1421-1442 of hPMR1 mRNA. The trilencer-27 universal scrambled negative control siRNA duplex (SR30004, OriGene) was used as the scrambled siRNA control. HEK293T cells were transfected twice at 24-h intervals 
with $50 \mathrm{nM}$ of each siRNA using DharmaFECT 1 (Thermo Scientific) following the manufacturer's protocol.

\section{Preparation of cytoplasmic extracts, sucrose gradients, and Western blot analysis}

Cells were harvested with trypsin, washed with ice-cold PBS, and transferred into a sterile $12 \times 75 \mathrm{~mm}$ polystyrene tube. For all experiments except polysome profile analysis, cells were collected by centrifugation for $1 \mathrm{~min}$ at $50 \mathrm{~g}$ and taken up in $100 \mu \mathrm{L}$ CER-I from the NE-PER kit (Pierce). Cytoplasmic extracts were prepared following the manufacturer's protocol except that gentle mixing was used in place of vortexing. These were either used directly for immunoprecipitation or polysome profile analysis or stored at $-80^{\circ} \mathrm{C}$ in buffer containing $10 \%$ glycerol. For polysome profile analysis, cells were lysed in buffer containing $15 \mathrm{mM}$ Tris $(\mathrm{pH}$ 7.5), $10 \mathrm{mM} \mathrm{MgCl}, 150 \mathrm{mM} \mathrm{KCl}, 500 \mu \mathrm{g} / \mathrm{mL}$ cycloheximide, $0.5 \%$ Triton $\mathrm{X}-100$, protease inhibitor cocktail (1:100 dilution from Sigma), phosphatase inhibitor cocktails 2 and 3 (1:100 dilution from Sigma), $2 \mathrm{mM}$ sodium orthovanadate, and $1 \mathrm{mM}$ phenylmethylsulfonyl fluoride. Three hundred microliters of cytoplasmic extract was layered onto $10 \%-50 \%$ linear gradients prepared in buffer containing $15 \mathrm{mM}$ Tris ( $\mathrm{pH} 7.5), 10 \mathrm{mM}$ $\mathrm{MgCl}_{2}, 150 \mathrm{mM} \mathrm{KCl}$, and $100 \mu \mathrm{g} / \mathrm{mL}$ cycloheximide. These were centrifuged at 209,627g in a Sorvall TH641 rotor for $3 \mathrm{~h}$ at $4^{\circ} \mathrm{C}$. Puromycin dissociation of polysomes was performed according to the method described by Yang and Schoenberg (2004) and was centrifuged as described above with the omission of cycloheximide from the buffer. One-half milliliter fractions were collected from the top using a Brandel gradient fractionator, and the absorbance at $254 \mathrm{~nm}$ was monitored continuously during fractionation. For Western blotting, samples were denatured in SDSsample buffer, separated on 10\% Mini-PROTEAN TGXTM gels (Bio-Rad Laboratories), and transferred onto Immobilon-P PVDF membrane (Millipore). Membranes were blocked with 5\% nonfat dry milk in TBS-T followed by incubation with primary antibody (1:500 dilution) and horseradish peroxidase-coupled secondary antibody (1:5000 dilution) in TBS-T containing 2\% BSA for antiPXDNL antibody or 5\% nonfat dry milk for all other antibodies. The peroxidase domain antibody variably cross-reacted with a 74$\mathrm{kDa}$ band that is not a product of PXDNL. This could be removed by preincubating extracts prior to electrophoresis for $30 \mathrm{~min}$ at $37^{\circ} \mathrm{C}$ in cell lysis buffer containing $5 \mathrm{mM}$ DTT followed by addition of $50 \mathrm{mM}$ iodoacetamide. Membranes were developed with ECL-plus (GE Healthcare) and visualized on blue ultra-X-ray film (GeneMate).

\section{Assays for reporter mRNA decay}

In the experiment in Figure 6B, tetracycline-inducible MCF-7 cells in antibiotic free medium were transfected with $0.75 \mu \mathrm{g}$ of pcDNA4/TO-Flag-hPMR1-Tev-biotin and $0.2 \mu \mathrm{g}$ each of plasmids expressing albumin and luciferase mRNA (Yang and Schoenberg 2004). Half of the cultures were treated with doxycycline to induce hPMR1 expression, and $50 \mu \mathrm{M}$ DRB (Sigma) was added $18 \mathrm{~h}$ later to block transcription. Cytoplasmic RNA was recovered from triplicate cultures at the time of DRB addition $(\mathrm{t}=0)$ and after 4 and $8 \mathrm{~h}$ in DRB-containing medium and was assayed by quantitative RT-PCR as described below. The experiment in Figure 7B was similar except that cells were transfected with $50 \mathrm{ng}$ each of plasmids expressing albumin and luciferase mRNA $16 \mathrm{~h}$ prior to adding DRB, and cytoplasmic RNA from triplicate cultures was recovered at the time of DRB addition $(t=0)$ and after $8 \mathrm{~h}$ in DRB-containing medium. In the experiment in Figure 7, C and D, cells were transfected twice at 24-h intervals with hPMR1 siRNA or the scrambled control. Fifty nanograms each of the albumin and luciferase plasmids was included in the second round of siRNA transfection, followed $16 \mathrm{~h}$ later by addition of DRB. The samples were harvested and assayed as described above.

\section{RNase protection, Q-PCR, and immunoprecipitation assays}

RNase protection assays were performed using a mixed albumin and luciferase probes according to the method described by Yang and Schoenberg (2004), and immunoprecipitation experiments were performed according to the method described by Peng and Schoenberg (2007). Q-PCR of albumin and luciferase mRNA used the primers 5'-CAAAGACCAGCCTTCAAACTC-3' (sense) and 5'-CACAGTTGAATGCTCTAAGCA-3' (antisense) for albumin mRNA, and 5'-AGATCCACAACCTTCGCTTC-3' (sense) and 5'-CTGAGGAGCCTTCAGGATTAC-3' (antisense) for luciferase mRNA that was used previously for RT-PCR according to the method described by Yang and Schoenberg (2004). Briefly, $1 \mu \mathrm{g}$ of cytoplasmic RNA was used to synthesize cDNA with Superscript III reverse transcriptase (Invitrogen) according to the manufacturer's instructions. Q-PCR was performed with $1 \mu \mathrm{L}$ of the cDNA in a $20 \mu \mathrm{L}$ reaction with SyBR green RT-PCR mix (SABiosciences) using an Illumina Eco system.

\section{SUPPLEMENTAL MATERIAL}

Supplemental material is available for this article.

\section{ACKNOWLEDGMENTS}

We thank Julie Dougherty for her help in packaging the lentiviral vectors, Juan Santiago-Torres for his help with creating tetracycline-inducible cell lines, and members of the Schoenberg laboratory for their helpful comments. We also thank Dr. Peter Kaiser (University of California, Irvine) for providing plasmid pFA6aHTB-hphMX4, which enabled the construction of plasmids expressing biotinylated tags. This work was supported by PHS grant GM038277 from the National Institute of General Medical Sciences.

Received November 11, 2011; accepted March 7, 2012.

\section{REFERENCES}

Bailey TL, Boden M, Buske FA, Frith M, Grant CE, Clementi L, Ren J, Li WW, Noble WS. 2009. MEME SUITE: tools for motif discovery and searching. Nucleic Acids Res 37: W202-W208.

Barnes T, Kim WC, Mantha AK, Kim SE, Izumi T, Mitra S, Lee CH. 2009. Identification of Apurinic/apyrimidinic endonuclease 1 (APE1) as the endoribonuclease that cleaves c-myc mRNA. Nucleic Acids Res 37: 3946-3958.

Cheng G, Salerno JC, Cao Z, Pagano PJ, Lambeth JD. 2008. Identification and characterization of VPO1, a new animal heme-containing peroxidase. Free Radic Biol Med 45: 1682-1694. 
Chernokalskaya E, DuBell AN, Cunningham KS, Hanson MN, Dompenciel RE, Schoenberg DR. 1998. A polysomal ribonuclease involved in the destabilization of albumin mRNA is a novel member of the peroxidase gene family. RNA 4: 1537-1548.

Cunningham KS, Hanson MN, Schoenberg DR. 2001. Polysomal ribonuclease 1 exists in a latent form on polysomes prior to estrogen activation of mRNA decay. Nucleic Acids Res 29: 1156-1162.

Dompenciel RE, Garnepudi VR, Schoenberg DR. 1995. Purification and characterization of an estrogen-regulated Xenopus liver polysomal nuclease involved in the selective destabilization of albumin mRNA. J Biol Chem 270: 6108-6118.

Eberle AB, Lykke-Andersen S, Muhlemann O, Jensen TH. 2009. SMG6 promotes endonucleolytic cleavage of nonsense mRNA in human cells. Nat Struct Mol Biol 16: 49-55.

Fejes-Toth K, Sotirova V, Sachidanandam R, Assaf G, Hannon GJ, Kapranov P, Foissac S, Willingham AT, Duttagupta R, Dumais E, et al. 2009. Post-transcriptional processing generates a diversity of 5'-modified long and short RNAs. Nature 457: 1028-1032.

Han D, Lerner AG, Vande Walle L, Upton JP, Xu W, Hagen A, Backes BJ, Oakes SA, Papa FR. 2009. IRE1 $\alpha$ kinase activation modes control alternate endoribonuclease outputs to determine divergent cell fates. Cell 138: 562-575.

Hollien J, Lin JH, Li H, Stevens N, Walter P, Weissman JS. 2009. Regulated Ire1-dependent decay of messenger RNAs in mammalian cells. J Cell Biol 186: 323-331.

Horikoshi N, Cong J, Kley N, Shenk T. 1999. Isolation of differentially expressed cDNAs from p53-dependent apoptotic cells: activation of the human homologue of the Drosophila peroxidasin gene. Biochem Biophys Res Commun 261: 864-869.

Huang H, Li L, Wu C, Schibli D, Colwill K, Ma S, Li C, Roy P, Ho K, Songyang Z, et al. 2008. Defining the specificity space of the human SRC homology 2 domain. Mol Cell Proteomics 7: 768-784.

Huntzinger E, Kashima I, Fauser M, Sauliere J, Izaurralde E. 2008. SMG6 is the catalytic endonuclease that cleaves mRNAs containing nonsense codons in metazoan. RNA 14: 2609-2617.

Kapranov P. 2009. From transcription start site to cell biology. Genome Biol 10: 217. doi: 10.1186/gb-2009-10-4-217.

Karginov FV, Cheloufi S, Chong MMW, Start A, Smith AD, Hannon GJ. 2010. Diverse endonucleolytic cleavage sites in the mammalian transcriptome depend on microRNAs, Drosha and additional nucleases. Mol Cell 38: 781-788.

Matsushita K, Takeuchi O, Standley DM, Kumagai Y, Kawagoe T, Miyake T, Satoh T, Kato H, Tsujimura T, Nakamura H, et al. 2009. $\mathrm{Zc} 3 \mathrm{~h} 12 \mathrm{a}$ is an RNase essential for controlling immune responses by regulating mRNA decay. Nature 458: 1185-1190.

Mercer TR, Dinger ME, Bracken CP, Kolle G, Szubert JM, Korbie DJ, Askarian-Amiri ME, Gardiner BB, Goodall GJ, Grimmond SM, et al. 2010. Regulated post-transcriptional RNA cleavage diversifies the eukaryotic transcriptome. Genome Res 20: 1639-1650.

Mizgalska D, Wegrzyn P, Murzyn K, Kasza A, Koj A, Jura J, Jarzab B, Jura J. 2009. Interleukin-1-inducible MCPIP protein has structural and functional properties of RNase and participates in degradation of IL-1 $\beta$ mRNA. FEBS J 276: 7386-7399.
Murray EL, Schoenberg DR. 2007. A+U-rich instability elements differentially activate $5^{\prime}-3^{\prime}$ and $3^{\prime}-5^{\prime}$ mRNA decay. Mol Cell Biol 27: 2791-2799.

Nechama M, Peng Y, Bell O, Briata P, Gherzi R, Schoenberg DR, Naveh-Many T. 2009. KSRP-PMR1-exosome association determines parathyroid hormone mRNA levels and stability in transfected cells. BMC Cell Biol 10: 70. doi: 10.1186/1471-2121-10-70.

Parker R, Sheth U. 2007. P bodies and the control of mRNA translation and degradation. Mol Cell 25: 635-646.

Pastori RL, Moskaitis JE, Schoenberg DR. 1991. Estrogen-induced ribonuclease activity in Xenopus liver. Biochemistry 30: 10490-10498.

Peng Y, Schoenberg DR. 2007. c-Src activates endonuclease-mediated mRNA decay. Mol Cell 25: 779-787.

Peng Y, Murray EL, Sarkar M, Liu X, Schoenberg DR. 2009. The cytoskeleton-associated Ena/VASP proteins are unanticipated partners of the PMR1 mRNA endonuclease. RNA 15: 576-587.

Peterfi Z, Donko A, Orient A, Sum A, Prokai A, Molnar B, Vereb Z, Rajnavolgyi E, Kovacs KJ, Muller V, et al. 2009. Peroxidasin is secreted and incorporated into the extracellular matrix of myofibroblasts and fibrotic kidney. Am J Pathol 175: 725-735.

Plessy C, Bertin N, Takahashi H, Simone R, Salimullah M, Lassmann T, Vitezic M, Severin J, Olivarius S, Lazarevic D, et al. 2010. Linking promoters to functional transcripts in small samples with nanoCAGE and CAGEscan. Nat Methods 7: 528-534.

Schoenberg DR. 2011. Mechanisms of endonuclease-mediated mRNA decay. Wiley Interdisc. Rev.: RNA 2: 582-600.

Schoenberg DR, Maquat LE. 2009. Re-capping the message. Trends Biochem Sci 34: 435-442.

Schoenberg DR, Maquat LE. 2012. Regulation of cytoplasmic mRNA decay. Nat Rev Genet 13: 246-259.

Suzuki HI, Arase M, Matsuyama H, Choi YL, Ueno T, Mano H, Sugimoto K, Miyazono K. 2011. MCPIP1 ribonuclease antagonizes Dicer and terminates microRNA biogenesis through precursor microRNA degradation. Mol Cell 44: 424-436.

Tagwerker C, Flick K, Cui M, Guerrero C, Dou Y, Auer B, Baldi P, Huang L, Kaiser P. 2006. A tandem affinity tag for two-step purification under fully denaturing conditions: application in ubiquitin profiling and protein complex identification combined with in vivo cross-linking. Mol Cell Proteomics 5: 737-748.

Tomecki R, Dziembowski A. 2010. Novel endoribonucleases as central players in various pathways of eukaryotic RNA metabolism. RNA 16: $1692-1724$.

Yang F, Schoenberg DR. 2004. Endonuclease-mediated mRNA decay involves the selective targeting of PMR1 to polyribosome-bound substrate mRNA. Mol Cell 14: 435-445.

Yang F, Peng Y, Schoenberg DR. 2004. Endonuclease-mediated mRNA decay requires tyrosine phosphorylation of polysomal ribonuclease 1 (PMR1) for the targeting and degradation of polyribosome-bound substrate mRNA. J Biol Chem 279: 48993-49002.

Zamocky M, Jakopitsch C, Furtmuller PG, Dunand C, Obinger C. 2008. The peroxidase-cyclooxygenase superfamily: Reconstructed evolution of critical enzymes of the innate immune system. Proteins 72: 589-605. 

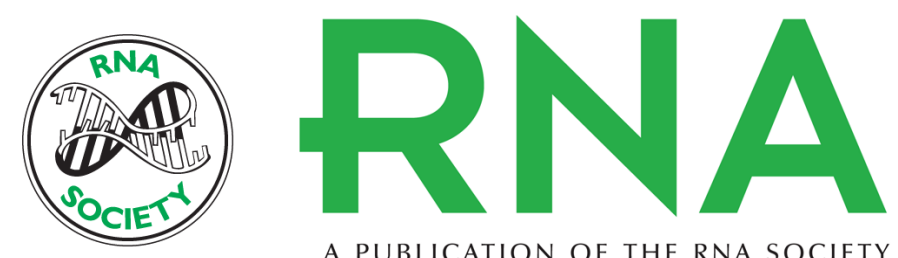

A PUBLICATION OF THE RNA SOCIETY

\section{Identification of the human PMR1 mRNA endonuclease as an alternatively processed product of the gene for peroxidasin-like protein}

Shan-Qing Gu, Baskar Bakthavachalu, Joonhee Han, et al.

RNA 2012 18: 1186-1196 originally published online April 27, 2012

Access the most recent version at doi:10.1261/rna.031369.111

Supplemental Material

References

License

Email Alerting Service
http://rnajournal.cshlp.org/content/suppl/2012/04/05/rna.031369.111.DC1

This article cites 35 articles, 11 of which can be accessed free at: http://rnajournal.cshlp.org/content/18/6/1186.full.html\#ref-list-1

Receive free email alerts when new articles cite this article - sign up in the box at the top right corner of the article or click here.

To subscribe to RNA go to:

http://rnajournal.cshlp.org/subscriptions 